\title{
Impact of a Food-grade Cationic Biopolymer (E-Polylysine) on the Digestion of Emulsified Lipids: In Vitro Study
}

\section{Cynthia Lyliam Lopez-Pena ${ }^{1}$ and David Julian McClements ${ }^{1,2}$}

\author{
${ }^{1}$ Department of Food Science, University of Massachusetts, Amherst, Massachusetts \\ 01003, United States \\ ${ }^{2}$ Department of Biochemistry, Faculty of Science, King Abdulaziz University, P. O.
}

Journal: Food Research International

Submitted: April 2015

Contact Information: David Julian McClements, Department of Food Science, University of Massachusetts, Amherst, Massachusetts 01003, United States; Tel 413545 1019; Fax 413545 1262; email mcclements@ foodsci.umass.edu 


\begin{abstract}
$\varepsilon$-Polylysine ( $\varepsilon$-PL) is a cationic biopolymer that may be used as a food ingredient because of its strong antimicrobial activity and potential to inhibit pancreatic lipase. We examined the effect of polylysine on the digestion of corn oil-in-water emulsions stabilized by either a natural anionic surfactant (quillaja saponin) or a synthetic non-ionic surfactant (Tween 20). Emulsions were prepared using high pressure homogenization (microfluidization) and then subjected to in vitro digestion in the absence or presence of polylysine at the maximum level allowed in foods by the FDA. Samples were characterized before and after in vitro digestion using electrophoresis, confocal microscopy, and static light scattering. The presence of polylysine decreased the hydrolytic activity of pancreatic lipase by around 53\% and $28 \%$ in the Tween 20 - and saponin-stabilized emulsions, respectively. The lipase-inhibiting properties of cationic polylysine were attributed to its electrostatic interaction with anionic components, such as bile salts, free fatty acids, or digestive enzymes. These results have important implications for the incorporation of polylysine into food systems, particularly those containing lipophilic nutrients.
\end{abstract}

Keywords: polylysine; lipids; digestion; emulsions; nanoemulsions; lipase inhibition 


\section{Introduction}

$\varepsilon$-Polylysine ( $\varepsilon$-PL) is an appealing antimicrobial agent for utilization within the food industry due to its natural origin and its strong antimicrobial activity against a wide range of Gram (+) and Gram (-) bacteria, yeasts, molds, and bacteriophages (El-Sersy, Abdelwahab, Abouelkhiir, Abou-Zeid, \& Sabry, 2012; Moschonas et al., 2012; Shima, Matsuoka, Iwamoto, \& Sakai, 1984; Yu, Huang, \& Huang, 2010; Zhou et al., 2011). The Food and Drug Administration (FDA) has deemed it as a Generally Regarded As Safe (GRAS) food ingredient, and has approved its use in a variety of food systems including alcoholic and non-alcoholic beverages, pastries, meats, soup, dairy products, fruit and vegetables products, and pasta (Food and Drug Administration, 2011).

The antimicrobial efficacy of $\varepsilon$-PL has been attributed to its high cationic charge density (Blin, Purohit, Leprince, Jouenne, \& Glinel, 2011; Brogden, 2005; Shima et al., 1984). However, the cationic nature of polylysine also limits its widespread use as a food ingredient because of its tendency to interact with anionic components in food matrices, which can decrease its antimicrobial efficacy and cause undesirable turbidity and precipitation. In addition, cationic polylysine may interact with anionic biopolymers in the saliva and mucus coating the mouth, which may lead to bitterness or astringency (Asker, Weiss, \& McClements, 2011; Chang, McLandsborough, \& McClements, 2011b; Lopez-Pena \& McClements, 2014). These problems may be overcome by forming weak electrostatic complexes between polylysine and anionic polysaccharides, such as pectin or gum arabic (Chang, McLandsborough, \& McClements, 2011a, 2012; Chang et al., 2011b; Chang, McLandsborough, \& McClements, 2014; Lopez-Pena \& McClements, 
2014). These electrostatic complexes have been shown to maintain the antimicrobial activity of polylysine, while inhibiting precipitation and sediment formation. However, another potential challenge that might limit the widespread application of $\varepsilon$-PL as a food ingredient is associated with its ability to participate in electrostatic interactions with bile salts and other anionic components in the small intestine, which may interfere with lipid digestion and absorption (Kido et al., 2003; Tsujita et al., 2003; Tsujita, Takaichi, Takaku, Aoyama, \& Hiraki, 2006). These interactions could lead to the malabsorption of lipophilic nutrients, vitamins, and nutraceuticals from food.

After ingestion, foods pass through the different regions of the gastrointestinal tract (GIT) where they are exposed to stresses, flow profiles, enzyme activities, salts, surface active substances, and $\mathrm{pH}$ changes, which aid in the digestion and absorption of nutrients and other bioactive substances (Basit, 2005). The three main intestinal components that facilitate lipid digestion and absorption are pancreatic lipase, bile salts, and phospholipids (Duan, 2000). Lipases in the mouth, stomach, and small intestine convert ingested triacylglycerols into free fatty acids and monoglycerides, with most of the digestion and absorption occurring in the small intestine (Lairon, 2009; Mackie, 2012; McClements \& Li, 2010c; McClements, 2007; Singh, Ye, \& Horne, 2009). Bile salts and phospholipids also play a number of key roles: (i) they help emulsify lipids by adsorbing to lipid droplet surfaces and forming a protective layer; (ii) they displace some of the original emulsifiers from the lipid droplet surfaces thereby altering interfacial composition in a manner that favors lipase adsorption; and (iii) they participate in the formation of mixed micelles that can solubilize free fatty acids and transport them to the epithelium cells (Hismiogullari, Bozdayi, \& Rahman, 2007). The free fatty acids and 
monoacylglycerols formed due to lipid digestion are incorporated into mixed micelles, transported across the mucus layer covering the intestinal wall, absorbed by intestinal microvilli, and then eventually enter the bloodstream (Kido et al., 2003).

As mentioned previously, research has shown that polylysine may inhibit lipid digestion. This phenomenon is proposed to be due to the electrostatic interaction between cationic $\varepsilon$-PL and anionic bile salts and phospholipids through three main mechanisms: (i) $\varepsilon$-PL binding to these digestive components, thereby preventing them from adsorbing to lipid droplet surfaces; (ii) $\varepsilon$-PL forming a cationic coat around anionic lipid droplets, thus preventing lipase from coming into close proximity to the lipids; (iii) $\varepsilon$-PL binding to bile salts and phospholipids, consequently retarding the formation of mixed micelles capable of solubilizing and transporting digested lipids (Kido et al., 2003).

Because a decrease in lipid digestion may adversely affect the absorption of essential lipophilic nutrients and nutraceuticals (Li \& McClements, 2011; Porter, Trevaskis, \& Charman, 2007), it is important to understand the possible impact of the incorporation of $\varepsilon$-PL into food products on lipid digestion. Previous studies that have shown the lipase-inhibiting properties of $\varepsilon$-PL have typically been carried out using $\varepsilon$-PL concentrations that far exceed the maximum levels approved by the FDA for food use. Additionally, these studies have used homogenization methods and emulsifiers that are not widely used in the food industry (Kido et al., 2003; Tsujita et al., 2003; Tsujita et al., 2006). The objective of the current study was therefore to test whether $\varepsilon$-PL used at the highest permitted concentration allowed by the FDA (Food and Drug Administration, 2011) has an impact on lipid digestion in emulsion-based delivery systems stabilized by food-grade ionic or non-ionic surfactants. It should be noted that previous studies have 
shown that other cationic biopolymers, such as chitosan, may interfere with the lipid digestion process by binding to lipid droplets, fatty acids, or bile salts (Beysseriat, Decker, \& McClements, 2006; Klinkesorn \& McClements, 2009). One might therefore expect polylysine to have a similar impact.

\section{Materials and Methods}

\subsection{Emulsion Preparation and Optimization}

A $5 \mathrm{mM}$ phosphate buffer was prepared by dissolving $1.1676 \mathrm{~g}$ of sodium phosphate monobasic (Sigma Aldrich, St. Louis, Missouri, lot BCBB2118) and $3.0932 \mathrm{~g}$ of sodium phosphate monobasic (Sigma Aldrich, St. Louis Missouri, lot 129K0053) to a final volume of 4 l. Emulsions were prepared by combining an oil phase $(4 \% \mathrm{w} / \mathrm{w})$ with an aqueous phase $(96 \% \mathrm{w} / \mathrm{w})$. The oil phase consisted of a commercial food-grade corn oil (Mazola, ACH Food Companies, London, England). The aqueous phase was composed of $5 \mathrm{mM}$ phosphate buffer ( $\mathrm{pH}$ 7) containing either non-ionic surfactant $(0.03$ $1.5 \%$ w/w Tween 20$)$ or anionic surfactant (0.05-0.95\% quillaja saponin). Tween 20 was acquired from Acros Organics (Hampton, New Hampshire, lot 091M1417V), while the quillaja saponin was donated in the form of Q-Naturale by Ingredion (Westchester, IL, lot QEU-151112-01, 14\% purity). $\varepsilon$-Polylysine was purchased from Wilshire Technologies (Princeton, NJ). The $\mathrm{pH}$ of the final systems was adjusted to a final value of 7.0 by adding either hydrochloric acid or sodium hydroxide at varying concentrations. 
A coarse emulsion was first prepared by mixing the oil and the aqueous phases with a high-shear blender (Bamix ${ }^{\circledR}$ Basic, Mettlen, Switzerland) at 7,000 rpm for 2 minutes. The resulting course emulsion was then passed five times through a microfluidizer (M-110P, Microfluidics, Westwood, MA) at 9,000 psi to produce a fine emulsion.

\subsection{In vitro Digestion}

In vitro digestions simulating only the small intestine stage were carried out by modifying the protocol established by $\mathrm{Li}, \mathrm{Hu}$, and McClements (2011).

Solutions for this experiment were prepared as follows. Bile salts (Sigma Aldrich, St. Louis, Missouri, lot 031M0106V) were prepared by dissolving $0.1875 \mathrm{~g}$ in $3.5 \mathrm{ml}$ of phosphate buffer $\mathrm{pH} 7.0$ and stirring overnight. A salt stock solution was produced by diluting $16.44 \mathrm{~g}$ of sodium chloride dihydrate (Fisher Scientific, Hampton, New Hampshire, lot 111354) and $2.57 \mathrm{~g}$ of calcium chloride (Sigma Aldrich, St. Louis, Missouri, lot 39H0085) in double-distilled water to a final volume of $75 \mathrm{ml}$. Lipase was prepared by dissolving $0.06 \mathrm{~g}$ of the powdered enzyme (Sigma Aldrich, St. Louis, Missouri, lot SLBC9250V) in $2.5 \mathrm{ml}$ of $5 \mathrm{mM}$ phosphate buffer, followed by stirring for 30 minutes. The enzyme was used immediately after its preparation.

For the systems without polylysine, $18.75 \mathrm{ml}$ of the prepared emulsions were diluted with $11.25 \mathrm{ml}$ of $5 \mathrm{mM}$ phosphate buffer. For systems containing polylysine, $18.75 \mathrm{ml}$ of sample were diluted with $11.25 \mathrm{ml}$ of a solution containing $0.16 \%(\mathrm{w} / \mathrm{w})$ polylysine (Willshire Technologies, Princeton, New Jersey, lot 20130228) in $5 \mathrm{mM}$ phosphate buffer, $\mathrm{pH}$ 7. When the emulsion was combined with the polylysine solutions, the final oil concentration for each system was $2.5 \%(\mathrm{w} / \mathrm{w})$ corn oil, and 2 ppm polylysine. 
The diluted emulsion sample was combined with the bile salts, stock salt solution, and lipase to simulate the digestion process in the small intestine. The rate and extent of lipid digestion was monitored using an automated $\mathrm{pH}$ Stat titration method (857 Titrando, Metrohm USA, Riverview, Florida). The principle behind this method is quantifying the lipase-mediated release of free fatty acids (FFA) from the triacylglycerols that make up the oil system. The sample is dispersed in a solution containing specific amounts of digestive components including bile salts, lipase, sodium chloride, and calcium chloride. As the lipase exerts its enzymatic activity over the oil present, FFAs are released. This production of FFAs causes a decrease in $\mathrm{pH}$, which is monitored by the automated titration unit; as the $\mathrm{pH}$ changes, the instrument automatically titrates sodium hydroxide solution to maintain it at $\mathrm{pH}$ 7.0. The amount of sodium hydroxide that was utilized throughout the digestion simulation is recorded versus time, and the percentage of FFA released can be calculated utilizing the equation below (Li et al., 2011):

$$
\% F F A=100 \times \frac{V_{\text {NaOH }} \times M_{N a O H} \times m_{\text {lipid }}}{w_{\text {lipid }} \times 2}
$$

Where \%FFA is the percentage of released FFAs; $\mathrm{V}_{\mathrm{NaOH}}$ is the volume of titrant in liters; $\mathrm{M}_{\mathrm{NaOH}}$ is the molarity of the sodium hydroxide solution used; $\mathrm{m}_{\text {lipid }}$ is the molecular weight of the oil used; and $\mathrm{w}_{\text {lipid }}$ is the weight of the oil in the digestion system in grams. 


\subsection{Particle Characterization}

The resulting emulsions and digestion products were characterized by measuring particle size (Malvern Mastersizer 2000, Malvern Instrumentst, Worcestershier, United Kingdom), $\zeta$-potential (Malvern Zetasizer ZS, Malvern Instruments, Worcestershire, United Kingdom), and confocal microscopy (Nikon D-Eclipse C1 80i, Nikon, Melville, NY) immediately after the emulsions were produced and after the conclusion of the

digestion process. The experimental protocols used for each of these methods have been described in detail elsewhere (Salvia-Trujillo, Qian, Martin-Belloso, \& McClements, 2013).

\subsection{Data Analysis}

All experiments were performed in triplicate. The results were then reported as averages and standard deviations of these measurements.

\section{Results and Discussion}

\subsection{Initial Emulsion Properties}

Initially, we aimed to produce oil-in-water emulsions that had relatively low concentrations of free surfactant in the aqueous phase, so as to prevent any interactions between $\varepsilon$-polylysine and excess surfactant that might interfere with data interpretation. Emulsions were therefore prepared using fixed homogenization conditions (oil content, homogenization pressure, and number of passes), but increasing concentrations of surfactants in the aqueous phase (Tcholakova, Denkov, \& Danner, 2004; Tcholakova, Denkov, Sidzhakova, Ivanov, \& Campbell, 2003). At low surfactant concentrations, there is insufficient surfactant to coat all of the small droplets formed within the 
homogenizer, and so the droplet size is mainly determined by the total amount of surfactant present. In this "surfactant-limited" regime, the mean particle diameter decreases with increasing surfactant concentration, and there is a relatively low concentration of free surfactant in the aqueous phase. At higher surfactant concentrations, the mean particle diameter remains relatively constant as the surfactant level is increased because the particle size is limited by the homogenization device, rather than the amount of surfactant present. In this "homogenizer-limited" regime, the amount of free surfactant in the aqueous phase increases as the total amount of surfactant in the system increases. Consequently, it is important to be within the surfactant-limited regime so as to reduce the amount of free surfactant present.

The influence of surfactant concentration on the mean particle diameter for emulsions prepared using either Tween 20 (T20) or quillaja saponin (QN) were therefore measured (Figures 1 and 2). In general, the dependence of the particle size on surfactant concentration was similar for both surfactants: initially there was a decrease in mean droplet diameter with increasing surfactant, followed by a leveling off. The optimal surfactant concentration was taken to be the value at which there was little further decrease in droplet size with increasing surfactant concentration, and the emulsion remained stable for at least 24 hours after production, i.e., there was no significant change in mean particle diameter or particle size distribution (Figure 3a). For T20 this value was $0.30 \%$ surfactant $\left(d_{43}=276 \mathrm{~nm}\right)$, whereas for QN it was $0.35 \%$ surfactant $\left(d_{32}=219 \mathrm{~nm}\right)$. Consequently, these surfactant concentrations were used in the subsequent experiments. The fact that the emulsions stabilized by QN had a smaller mean diameter than those 
stabilized by T20 may have been due to faster surfactant adsorption, greater reduction in interfacial tension, and/or better protection against recoalescence in the homogenizer.

\subsection{Particle Size Analysis and Confocal Microscopy}

Initially, we measured the influence of emulsifier type and $\varepsilon$-PL addition on changes in the particle size and microstructure of the emulsions before and after digestion. Prior to digestion and polylysine addition, both types of emulsions contained relatively small droplets $\left(\mathrm{d}_{43}<300 \mathrm{~nm}\right)$ and had monomodal particle size distributions (Figures 3a,b,c).

In the absence of $\varepsilon$-PL, the mean diameter of the particles in the T20-emulsions increased appreciably after being subjected to in vitro digestion, with $\mathrm{d}_{43}$ changing from around 276 to $484 \mathrm{~nm}$ (Figure 3a). There was also a large increase in mean particle diameter after digestion for the QN-emulsions, with $\mathrm{d}_{43}$ changing from around 219 to $1695 \mathrm{~nm}$ (Figure 3a). For both emulsions, the particle size distribution was still mainly monomodal, but with evidence of a few larger particles (Figures 3b and 3c). In general, a change in particle size distribution of emulsions after digestion can be attributed to a number of phenomena, including droplet hydrolysis, coalescence, flocculation, formation of mixed micelles (micelles and vesicles), and formation of insoluble precipitates (such as calcium soaps) (Devraj et al., 2013; Williams et al., 2013). The difference in particle size measured after digestion for the two surfactants suggests that there were some differences in the structural properties of any remaining undigested lipid droplets or lipid digestion products (Li et al., 2011). These results were supported by the microstructure images obtained through confocal microscopy, which showed that both emulsions 
contained relatively small evenly dispersed lipid droplets before digestion, but that they contained some relatively large particles after digestion (Figure 4).

The addition of polylysine to the T20-emulsions prior to digestion did not alter the mean particle diameter (Figure 3a) or particle size distribution (Figure 3c) of the droplets, which can be attributed to the fact that cationic $\varepsilon$-PL did not interact strongly with the non-ionic surfactant coated lipid droplets. Presumably, there was no strong electrostatic attraction between the polylysine and the droplet surfaces, but there was a strong steric repulsion between the polylysine and the neutral polymeric head groups of this surfactant. On the other hand, there was a large increase in the mean particle diameter (Figure 3a) and evidence of a population of large particles in the particle size distribution (Figure 3c) for the T20-emulsions containing $\varepsilon$-PL after digestion, which suggested that the cationic biopolymer promoted extensive aggregation of the anionic undigested lipid droplets and/or mixed micelles. It should be noted that the composition and structure of the T20-emulsions will have been changed considerably after exposure to the simulated digestion conditions (McClements, Decker, \& Park, 2009; Singh et al., 2009). In particular, undigested lipid droplets and mixed micelles are likely to contain anionic fatty acids or bile salts at their surfaces, which would promote interactions with cationic biopolymers in the small intestine. 
Prior to digestion, the addition of polylysine to the QN-emulsions caused a large increase in mean particle diameter (Figures 3a and 3b), which can be attributed to bridging flocculation of the anionic droplets by the cationic biopolymer (Klinkesorn \& McClements, 2009). After digestion, the QN-emulsions containing polylysine also had very large particle sizes, indicating that they were highly flocculated. This effect can be attributed to the formation of electrostatic complexes between cationic $\varepsilon$-PL molecules and anionic species in the digesta, such as bile salts, phospholipids, free fatty acids, and undigested fat droplets.

Confocal microscopy images of the emulsions containing polylysine supported the light scattering results (Figure 4). Prior to digestion, the addition of polylysine to the T20-emulsions had little influence on their microstructure, but its addition to the QNemulsions promoted extensive droplet aggregation. After digestion, the emulsions contained spherical lipid particles, which may have been undigested fat droplets or vesicles formed by bile salts, phospholipids, and fatty acids (Singh et al., 2009).

\subsection{Micro-Electrophoresis Measurements}

In this section, we characterized changes in the surface charge of the two emulsions with and without $\varepsilon$-PL, as well as before and after digestion (Figure 5). As mentioned previously, these two surfactants were partly selected for this study because of their different surface characteristics, which would have been expected impact their interactions with $\varepsilon$-PL. Prior to digestion, the T20-emulsion containing no polylysine had a relatively low negative charge $(-5.8 \mathrm{mV})$, whereas the $\mathrm{QN}$-emulsion has a relatively high negative charge $(-63.2 \mathrm{mV})$. After digestion, the negative charge on the particles present in the T20-emulsions increased $(-74.5 \mathrm{mV})$ appreciably, which can be attributed 
to the presence of the anionic bile salts, phospholipids, and free fatty acids. The particles in the QN-emulsions were also highly negative after digestion $(-42.1 \mathrm{mV})$, but it was less negative that the emulsions prior to digestion. These results suggest that there were different kinds of structures formed in the digesta after lipase digestion of emulsions initially stabilized by Q-Naturale and Tween 20. It is possible that these amphiphilic molecules were able to be incorporated into micelles and vesicles ("mixed micelles") formed by lipid digestion, thereby altering their charge and structural characteristics ( $\mathrm{Li}$ et al., 2011; Singh et al., 2009).

Prior to digestion, the addition of polylysine to the T20-emulsions caused little change in the electrical characteristics of the droplets $(-6.2 \mathrm{mV})$ compared to the samples with no polylysine $(-5.8 \mathrm{mV})$. This suggests that polylysine did not interact strongly with the surfaces of the lipid droplets coated by the non-ionic surfactant. Conversely, the incorporation of polylysine into the QN-emulsions prior to digestion caused an appreciable change in their surface charge characteristics, with the $\zeta$-potential going from -63.2 to $-4.2 \mathrm{mV}$ upon addition of polylysine. This suggests that the cationic polylysine molecules adsorbed to the surfaces of the anionic QN-coated lipid droplets through electrostatic attraction, thereby partially neutralizing their charge, as has previously been reported for chitosan (Klinkesorn \& McClements, 2009). After digestion, the particles in the digesta resulting from the emulsions initially containing QN-coated lipid droplets and polylysine were highly negative $(-45 \mathrm{mV})$. This effect can be attributed to the relatively high concentration of anionic species after digestion, such as bile salts, phospholipids, and free fatty acids. Presumably, the high negative charge from these species dominated any positive charge from the polylysine. 


\subsection{Free Fatty Acid Formation during Lipolysis}

The main objective of this study was to assess the potential inhibitory action of polylysine on lipid digestion in the small intestine, and to determine the influence of initial surfactant type on this effect. A simulated small intestine (pH stat) method was therefore used to focus on the events occurring within this region of the GIT where the majority of lipid digestion normally occurs. In reality, an emulsion passes through the mouth and stomach before reaching the small intestine, which may cause alterations in the size and surface characteristics of the lipid droplets. Nevertheless, previous studies have shown that $\varepsilon$-PL is resistant to degradation by digestive enzymes in the upper gastrointestinal tract (Tsujita et al., 2006), and that lipid droplets coated by some small molecule surfactants do not undergo appreciable changes in their properties in the mouth and stomach regions (McClements et al., 2009; McClements \& Li, 2010c; McClements, 2007; Singh et al., 2009). Hence, the results obtained in this study should provide some valuable insights into the potential role of polylysine on lipid digestion in the small intestine. In future studies, it would be useful to examine the influence of polylysine on lipid digestion using a full gastrointestinal model: mouth, stomach, small intestine.

In general, the free fatty acid release profiles of the different systems exhibited a similar behavior: initially there was a rapid increase in FFAs released followed by a more gradual increase at longer times (Figure 6). However, the shape of the FFA release profiles depended on emulsifier type and $\varepsilon$-PL addition. In the absence of polylysine, the fraction of the lipid phase digested after 2 hours incubation was about $43 \%$ and $52 \%$ for the T20 and QN systems, respectively (Figure 6). The relatively low amount of FFAs released from the emulsions can be attributed to the relatively high level of lipids present 
in the initial samples, i.e., there were too many triacyglycerol molecules for the lipase to completely digest and/or for the mixed micelles to completely solubilize (Li et al., 2011). These experiments may therefore be more representative of conditions where a high fat load is ingested as part of a meal. The addition of $\varepsilon$-PL had a major impact on lipid digestion for both surfactant types leading to a decrease in the rate and extent of FFA production. For example, at the end of the 2 hour incubation period, the amount of FFAs released was around 21\% and 34\% for T20 and QN systems, respectively. These values correspond to a decrease of 53\% and 35\% in FFA production compared to the samples with no polylysine. The suppression of lipid digestion by $\varepsilon-\mathrm{PL}$ observed in this study is in agreement with that reported in earlier studies using different types of emulsions, where an inhibitory effect of up to 50\% was also reported (Kido et al., 2003; Tsujita et al., 2003; Tsujita et al., 2006; Tsujita \& Takaku, 2009).

There are a number of potential physicochemical mechanisms that may account for the observed suppression of lipid digestion in the presence of polylysine. Cationic polylysine may adsorb to the surface of anionic lipid droplets and form a protective coating that inhibits the ability of the lipase to interact with the lipids. Cationic polylysine may interact with anionic bile salts and phospholipids through electrostatic interactions thereby changing their functional properties. As mentioned in the introduction, bile salts and phospholipids play various roles in lipid digestion: they aid in lipid emulsification within the GIT; they alter lipid droplet surfaces in a manner that promotes lipase adsorption and activation; they aid in the solubilization and transport of lipid digestion products by forming mixed micelles (Kido et al., 2003). In this study, the lipid droplets were homogenized prior to entering the small intestine, and therefore the 
role of bile salts and phospholipids on emulsification is less important. However, the ability of $\varepsilon$-PL to induce droplet flocculation may alter the rate and extent of lipid digestion. Previous studies have shown that highly flocculated lipid droplets are digested more slowly than non-flocculated ones because of a reduction in the surface area of lipid phase directly exposed to the lipase molecules (Day et al., 2014). The adsorption of bile salts to oil droplet surfaces (and displacement of the original surfactants) is often critical to lipid digestion, as the formation of a bile-salt coated interface promotes lipase adsorption and activation (Aloulou et al., 2006; Borgström \& Brockman, 1984; Tsujita et al., 2007). Moreover, the activity of lipase is determined by the amount that adsorbs to the lipid droplet surfaces, rather than the total amount present in the system (Moreau, Moulin, Gargouri, Noel, \& Verger, 1991; Ransac et al., 1997; Tsujita et al., 2007). When no $\varepsilon-\mathrm{PL}$ is present, bile salts are free to interact with the oil droplets, establishing suitable conditions on the droplet surfaces for the subsequent adsorption and activation of lipase. However, when cationic polylysine is present, it interacts with the anionic bile salts and prevents them from coating the oil droplet surfaces, thereby altering lipase adsorption and activity (Tsujita et al., 2003; Tsujita et al., 2007).

When anionic surfactants (such as saponin) are present in an emulsion, the cationic polylysine molecules may interact with them as well as with the anionic bile salts. As a result, there may be more free bile salts available to interact with the lipid droplet surfaces and to form mixed micelles, which would promote digestion. The changes in $\zeta$ potential observed for both systems upon the introduction of $\varepsilon$-PL provided additional insights into the difference in the degree of lipase inhibition exhibited. Tsujita et al. (2003) have shown that for $\varepsilon$-PL to exhibit lipase-inhibiting behavior in an emulsion 
system with a fixed amount of bile salts, the positive charges contributed by $\varepsilon$-PL must surpass the negative charges in the system. The inclusion of a negative surfactant and subsequent increase in negative charge means that the system no longer complies with this premise, limiting the inhibitory effect of $\varepsilon$-PL.

\section{Conclusions}

There is interest in utilizing polylysine as a functional ingredient in foods and beverages because it is a natural antimicrobial. However, our results and those of other groups working with phospholipid-stabilized emulsions, suggest that this cationic biopolymer may interfere with the normal lipid digestion process due to its ability to interact with anionic components in the gastrointestinal tract, such as lipid droplets, free fatty acids, bile salts, and phospholipids. These interactions depend on surfactant type, and may have an impact on the absorption of lipophilic bioactive agents, such as oilsoluble vitamins or nutraceuticals. In particular, we found that polylysine reduced the extent of free fatty acid release from oil-in-water emulsions under simulated small intestine conditions, with the effects being greater for Tween 20 (non-ionic) than for QNaturale (anionic).

It should be noted that a highly simplified in vitro digestion method was utilized in this study that only focused on the events occurring within the small intestine region. This method was used as an initial screening tool to establish whether polylysine would interfere with the processes occurring the small intestine where the majority of lipid digestion and absorption normally occurs. In future studies, more sophisticated in vitro digestion models that include mouth, stomach and small intestine stages should be used, 
as well as in vivo studies using animals (McClements \& Li, 2010a; Minekus et al., 2014).

For this reason, we intend to use a more comprehensive in vitro digestion model and animal feeding studies to assess the potential influence of polylysine on lipid digestion and nutrient bioaccessibility in our future work.

\section{Acknowledgments}

This material is based upon work supported by the Cooperative State Research, Extension, Education Service, United State Department of Agriculture, Massachusetts Agricultural Experiment Station (Project No. 831) and by the United States Department of Agriculture, NRI Grants (2010-03459, 2011-03539, 2013-03795, 2011-67021, and 2014-67021). 


\section{References}

Aloulou, A., Rodriguez, J. A., Fernandez, S., van Oosterhout, D., Puccinelli, D., \& Carriere, F. (2006). Exploring the specific features of interfacial enzymology based on lipase studies. Biochimica Et Biophysica Acta-Molecular and Cell Biology of Lipids, 1761 (9), 995-1013.

Asker, D., Weiss, J., \& McClements, D. J. (2011). Formation and Stabilization of Antimicrobial Delivery Systems Based on Electrostatic Complexes of Cationic-Non-ionic Mixed Micelles and Anionic Polysaccharides. Journal of Agricultural and Food Chemistry, 59 (3), 1041-1049.

Basit, A. W. (2005). Advances in colonic drug delivery. Drugs, 65 (14), 1991-2007.

Beysseriat, M., Decker, E. A., \& McClements, D. J. (2006). Preliminary study of the influence of dietary fiber on the properties of oil-in-water emulsions passing through an in vitro human digestion model. Food Hydrocolloids, 20 (6), 800809.

Blin, T., Purohit, V., Leprince, J., Jouenne, T., \& Glinel, K. (2011). Bactericidal Microparticles Decorated by an Antimicrobial Peptide for the Easy Disinfection of Sensitive Aqueous Solutions. Biomacromolecules, 12 (4), 12591264.

Borgström, B., \& Brockman, H. L. (1984). Lipases. New York, N.Y.: Elsevier.

Brogden, K. A. (2005). Antimicrobial peptides: Pore formers or metabolic inhibitors in bacteria? Nature Reviews Microbiology, 3 (3), 238-250.

Chang, Y., McLandsborough, L., \& McClements, D. J. (2011a). Interactions of a Cationic Antimicrobial (epsilon-Polylysine) with an Anionic Biopolymer (Pectin): An Isothermal Titration Calorimetry, Microelectrophoresis, and Turbidity Study. Journal of Agricultural and Food Chemistry, 59 (10), 55795588.

Chang, Y., McLandsborough, L., \& McClements, D. J. (2012). Cationic Antimicrobial (epsilon-Polylysine)-Anionic Polysaccharide (Pectin) Interactions: Influence of Polymer Charge on Physical Stability and Antimicrobial Efficacy. Journal of Agricultural and Food Chemistry, 60 (7), 1837-1844.

Chang, Y. H., McLandsborough, L., \& McClements, D. J. (2011b). Physicochemical Properties and Antimicrobial Efficacy of Electrostatic Complexes Based on Cationic epsilon-Polylysine and Anionic Pectin. Journal of Agricultural and Food Chemistry, 59 (12), 6776-6782.

Chang, Y. H., McLandsborough, L., \& McClements, D. J. (2014). Antimicrobial delivery systems based on electrostatic complexes of cationic epsilon-polylysine and anionic gum arabic. Food Hydrocolloids, 35, 137-143.

Day, L., Golding, M., Xu, M., Keogh, J., Clifton, P., \& Wooster, T. J. (2014). Tailoring the digestion of structured emulsions using mixed monoglyceride-caseinate interfaces. Food Hydrocolloids, 36, 151-161. 
Devraj, R., Williams, H. D., Warren, D. B., Mullertz, A., Porter, C. J. H., \& Pouton, C. W. (2013). In vitro digestion testing of lipid-based delivery systems: Calcium ions combine with fatty acids liberated from triglyceride rich lipid solutions to form soaps and reduce the solubilization capacity of colloidal digestion products. International Journal of Pharmaceutics, 441 (1-2), 323-333.

Duan, R. D. (2000). Enzymatic aspects of fat digestion in the gastro- intestinal tract. In A. B. Christophe \& S. DeVriese (Eds.), Fat Digestion and Absorption (pp. 2546): AOCS Press.

El-Sersy, N. A., Abdelwahab, A. E., Abouelkhiir, S. S., Abou-Zeid, D. M., \& Sabry, S. A. (2012). Antibacterial and Anticancer activity of epsilon-poly-L-lysine (epsilon-PL) produced by a marine Bacillus subtilis sp. Journal of Basic Microbiology, 52 (5), 513-522.

Food and Drug Administration. (2011). Agency Response Letter GRAS Notice No. GRN 000336. In (Vol. 2014).

Hismiogullari, A. A., Bozdayi, A. M., \& Rahman, K. (2007). Biliary lipid secretion. Turkish Journal of Gastroenterology, 18 (2), 65-70.

Kido, Y., Hiramoto, S., Murao, M., Horio, Y., Miyazaki, T., Kodama, T., \& Nakabou, Y. (2003). epsilon-Polylysine inhibits pancreatic lipase activity and suppresses postprandial hypertriacylglyceridemia in rats. Journal of Nutrition, 133 (6), 1887-1891.

Klinkesorn, U., \& McClements, D. J. (2009). Influence of chitosan on stability and lipase digestibility of lecithin-stabilized tuna oil-in-water emulsions. Food Chemistry, 114 (4), 1308-1315.

Lairon, D. (2009). Digestion and Absorption of Lipids. In D. J. D. McClements, Eric A. (Ed.), Designing Functional Foods (pp. 68-93). Boca Raton, Florida: CRC Press.

Li, Y., Hu, M., \& McClements, D. J. (2011). Factors affecting lipase digestibility of emulsified lipids using an in vitro digestion model: Proposal for a standardised pH-stat method. Food Chemistry, 126 (2), 498-505.

Li, Y., \& McClements, D. J. (2011). Inhibition of lipase-catalyzed hydrolysis of emulsified triglyceride oils by low-molecular weight surfactants under simulated gastrointestinal conditions. European Journal of Pharmaceutics and Biopharmaceutics, 79 (2), 423-431.

Lopez-Pena, C. L., \& McClements, D. J. (2014). Optimizing delivery systems for cationic biopolymers: Competitive interactions of cationic polylysine with anionic $\kappa$-carrageenan and pectin. Food Chemistry, 153, 9-14.

Mackie, A. (2012). Interaction of Food Ingredient and Nutraceutical Delivery Systems With the Human Gastrointestinal Tract. In N. Garti \& D. J. McClements (Eds.), Encapsulation Technologies and Delivery Systems for Food Ingredients and Nutraceuticals. Cambridge, UK: Woodhead Publishing Limited.

McClements, D. J., Decker, E. A., \& Park, Y. (2009). Controlling Lipid Bioavailability through Physicochemical and Structural Approaches. Critical Reviews in Food Science and Nutrition, 49 (1), 48-67.

McClements, D. J., \& Li, Y. (2010a). Review of in vitro digestion models for rapid screening of emulsion-based systems. Food \& Function, 1 (1), 32-59. 
McClements, D. J., \& Li, Y. (2010c). Structured emulsion-based delivery systems: Controlling the digestion and release of lipophilic food components. Advances in Colloid and Interface Science, 159 (2), 213-228.

McClements, D. J. D., Eric A.; Park,Y. (2007). Physicochemical and structural aspects of lipid digestion. In D. J. McClements (Ed.), Understanding and Controlling the Microstructure of Complex Foods (pp. 483-503). Boca Raton, FL: CRC Press.

Minekus, M., Alminger, M., Alvito, P., Ballance, S., Bohn, T., Bourlieu, C., Carriere, F., Boutrou, R., Corredig, M., Dupont, D., Dufour, C., Egger, L., Golding, M., Karakaya, S., Kirkhus, B., Le Feunteun, S., Lesmes, U., Macierzanka, A., Mackie, A., Marze, S., McClements, D. J., Menard, O., Recio, I., Santos, C. N., Singh, R. P., Vegarud, G. E., Wickham, M. S. J., Weitschies, W., \& Brodkorb, A. (2014). A standardised static in vitro digestion method suitable for food - an international consensus. Food \& Function, 5 (6), 1113-1124.

Moreau, H., Moulin, A., Gargouri, Y., Noel, J. P., \& Verger, R. (1991). Inactivation of gastric and pancreatic lipases by diethyl para-nitrophenyl phosphate. Biochemistry, 30 (4), 1037-1041.

Moschonas, G., Geornaras, I., Stopforth, J. D., Wach, D., Woerner, D. R., Belk, K. E., Smith, G. C., \& Sofos, J. N. (2012). Activity of Caprylic Acid, Carvacrol, epsilonPolylysine and their Combinations against Salmonella in Not-Ready-to-Eat Surface-Browned, Frozen, Breaded Chicken Products. Journal of Food Science, 77 (7), M405-M411.

Porter, C. J. H., Trevaskis, N. L., \& Charman, W. N. (2007). Lipids and lipid-based formulations: optimizing the oral delivery of lipophilic drugs. Nature Reviews Drug Discovery, 6 (3), 231-248.

Ransac, S., Gargouri, Y., Marguet, F., Buono, G., Beglinger, C., Hildebrand, P., Lengsfeld, H., Hadvary, P., \& Verger, R. (1997). Covalent inactivation of lipases. In B. Rubin \& E. A. Dennis (Eds.), Lipases, Pt B: Enzyme Characterization and Utilization (Vol. 286, pp. 190-231).

Salvia-Trujillo, L., Qian, C., Martin-Belloso, O., \& McClements, D. J. (2013). Influence of particle size on lipid digestion and beta-carotene bioaccessibility in emulsions and nanoemulsions. Food Chemistry, 141 (2), 1472-1480.

Shima, S., Matsuoka, H., Iwamoto, T., \& Sakai, H. (1984). ANTIMICROBIAL ACTION OF EPSILON-POLY-L-LYSINE. Journal of Antibiotics, 37 (11), 1449-1455.

Singh, H., Ye, A. Q., \& Horne, D. (2009). Structuring food emulsions in the gastrointestinal tract to modify lipid digestion. Progress in Lipid Research, 48 (2), 92-100.

Tcholakova, S., Denkov, N. D., \& Danner, T. (2004). Role of surfactant type and concentration for the mean drop size during emulsification in turbulent flow. Langmuir, 20 (18), 7444-7458.

Tcholakova, S., Denkov, N. D., Sidzhakova, D., Ivanov, I. B., \& Campbell, B. (2003). Interrelation between drop size and protein adsorption at various emulsification conditions. Langmuir, 19 (14), 5640-5649.

Tsujita, T., Sumiyoshi, M., Takaku, T., Momsen, W. E., Lowe, M. E., \& Brockman, H. L. (2003). Inhibition of lipases by epsilon-polylysine. Journal of Lipid Research, 44 (12), 2278-2286. 
Tsujita, T., Takaichi, H., Takaku, T., Aoyama, S., \& Hiraki, J. (2006). Antiobesity action of epsilon-polylysine, a potent inhibitor of pancreatic lipase. Journal of Lipid Research, 47 (8), 1852-1858.

Tsujita, T., Takaichi, H., Takaku, T., Sawai, T., Yoshida, N., \& Hiraki, J. (2007). Inhibition of lipase activities by basic polysaccharide. Journal of Lipid Research, 48 (2), 358-365.

Tsujita, T., \& Takaku, T. (2009). Inhibition by epsilon-Polylysine of Fat Digestion in the Stomach and Intestine of Rats. Bioscience Biotechnology and Biochemistry, 73 (3), 536-542.

Williams, H. D., Trevaskis, N. L., Charman, S. A., Shanker, R. M., Charman, W. N., Pouton, C. W., \& Porter, C. J. H. (2013). Strategies to Address Low Drug Solubility in Discovery and Development. Pharmacological Reviews, 65 (1), 315-499.

Yu, H. L., Huang, Y. P., \& Huang, Q. R. (2010). Synthesis and Characterization of Novel Antimicrobial Emulsifiers from epsilon-Polylysine. Journal of Agricultural and Food Chemistry, 58 (2), 1290-1295.

Zhou, C. C., Li, P., Qi, X. B., Sharif, A. R. M., Poon, Y. F., Cao, Y., Chang, M. W., Leong, S. S. J., \& Chan-Park, M. B. (2011). A photopolymerized antimicrobial hydrogel coating derived from epsilon-poly-L-lysine. Biomaterials, 32 (11), 2704-2712. 


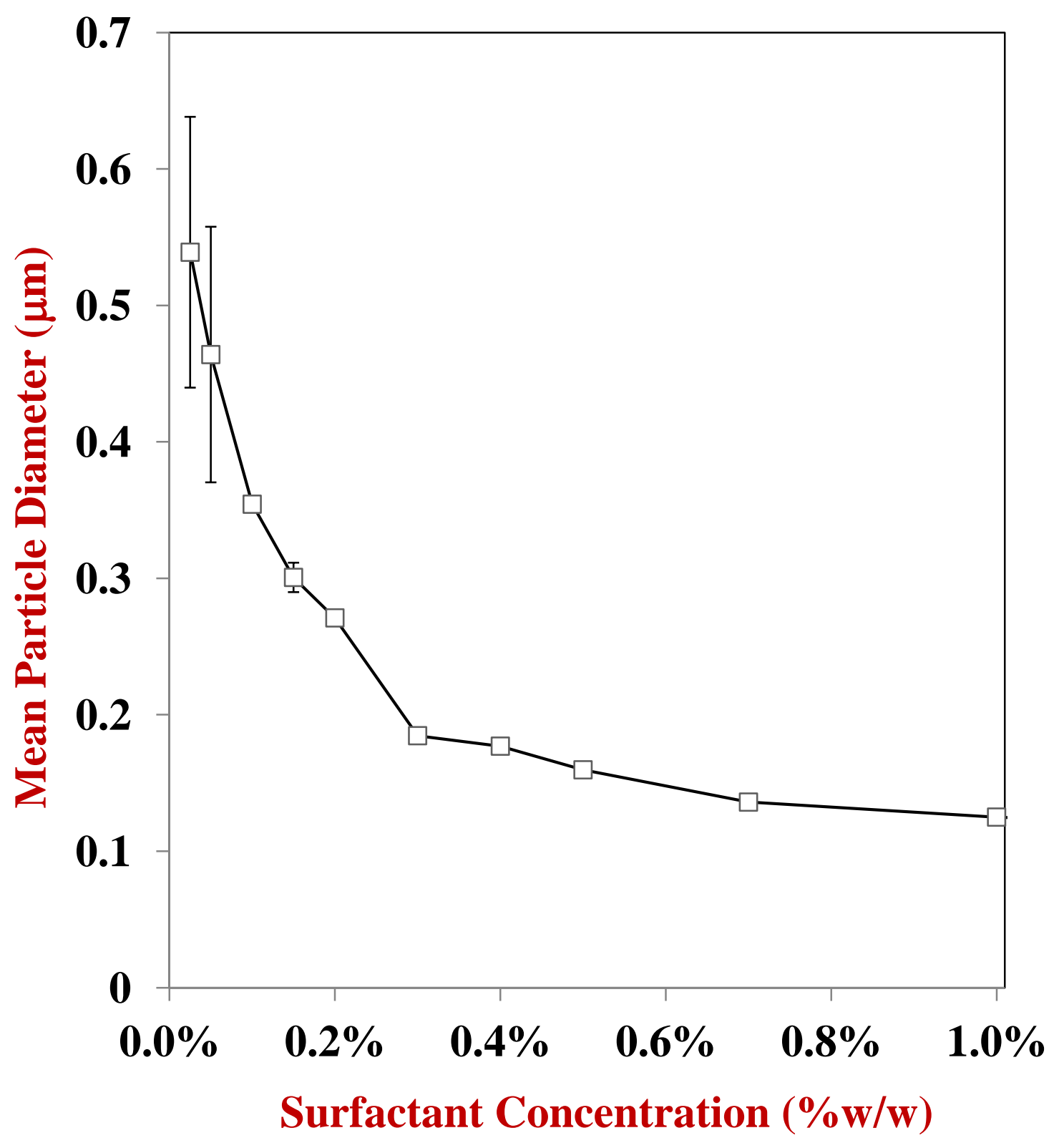

Figure 1. Influence of surfactant concentration on the mean particle diameter $\left(\mathrm{d}_{32}\right)$ of Tween 20-stabilized oil-in-water emulsions (4\% oil). 


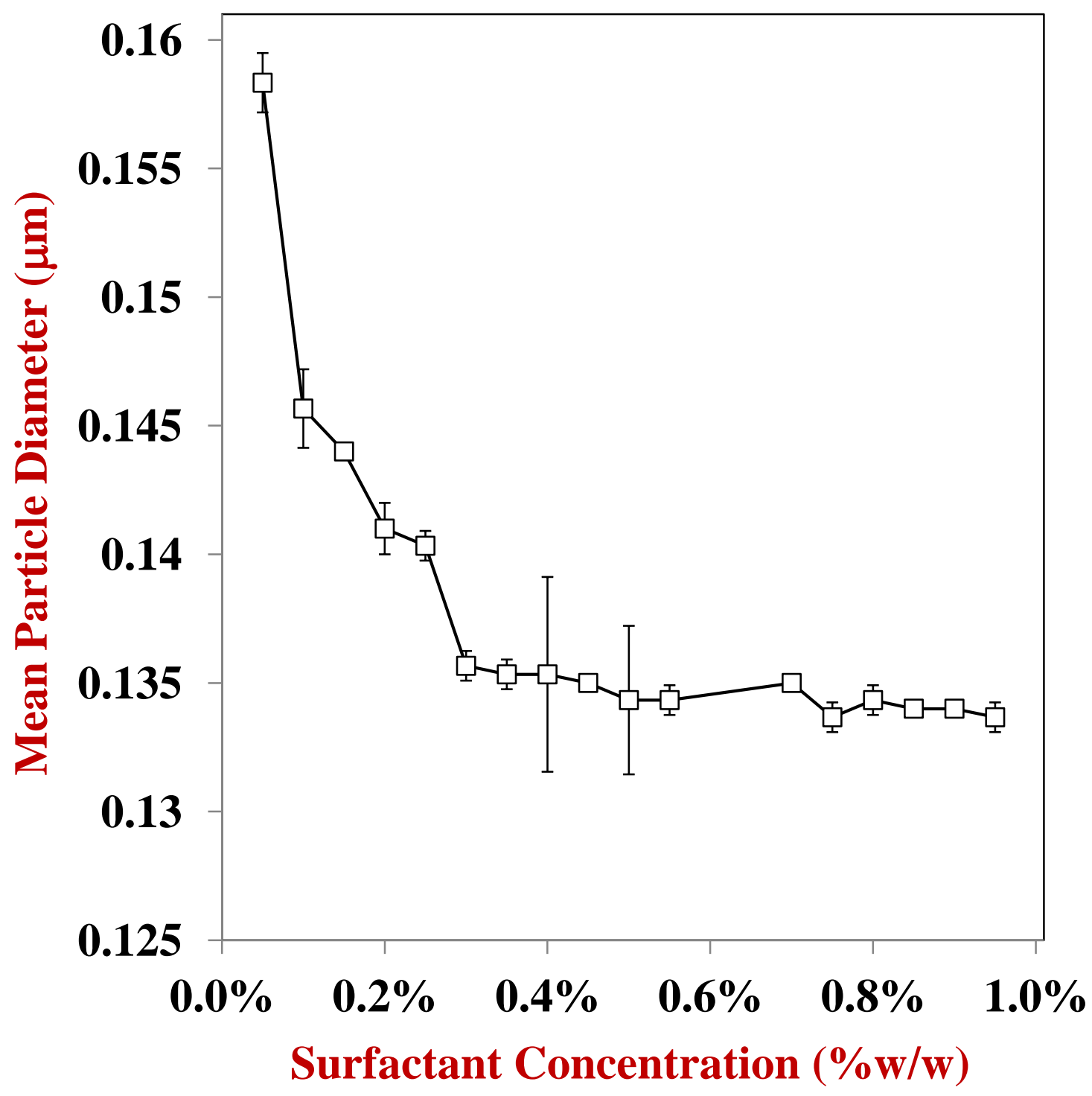

Figure 2. Influence of surfactant concentration on the mean particle diameter $\left(\mathrm{d}_{32}\right)$ of $\mathrm{Q}$ Naturale-stabilized oil-in-water emulsions ( $4 \%$ oil). 


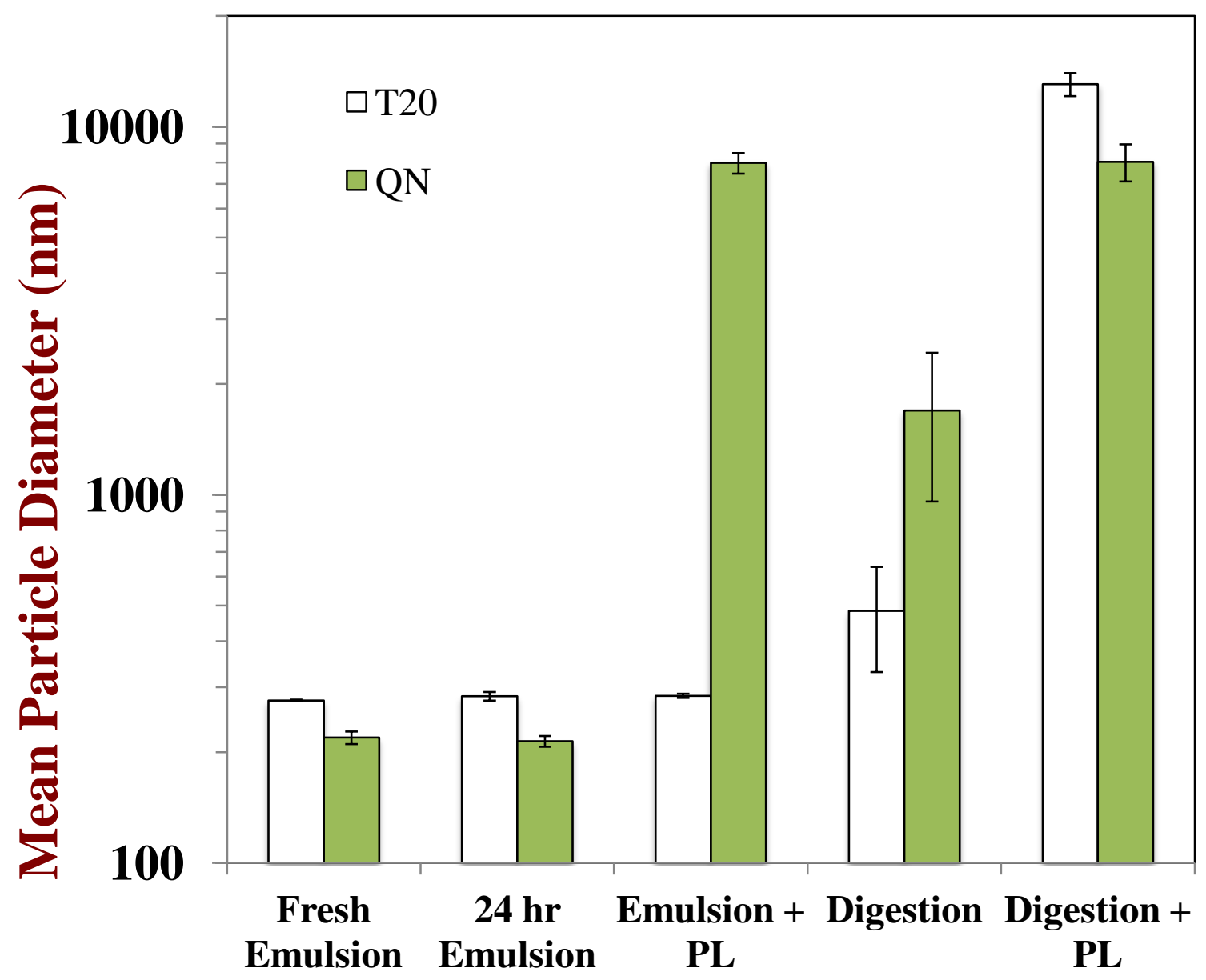

Figure 3a. Mean particle diameters $\left(\mathrm{d}_{43}\right)$ of the original and digested emulsions systems with and without $\varepsilon$-PL for both Tween 20 (T20) and Q-Naturale (QN) systems. 


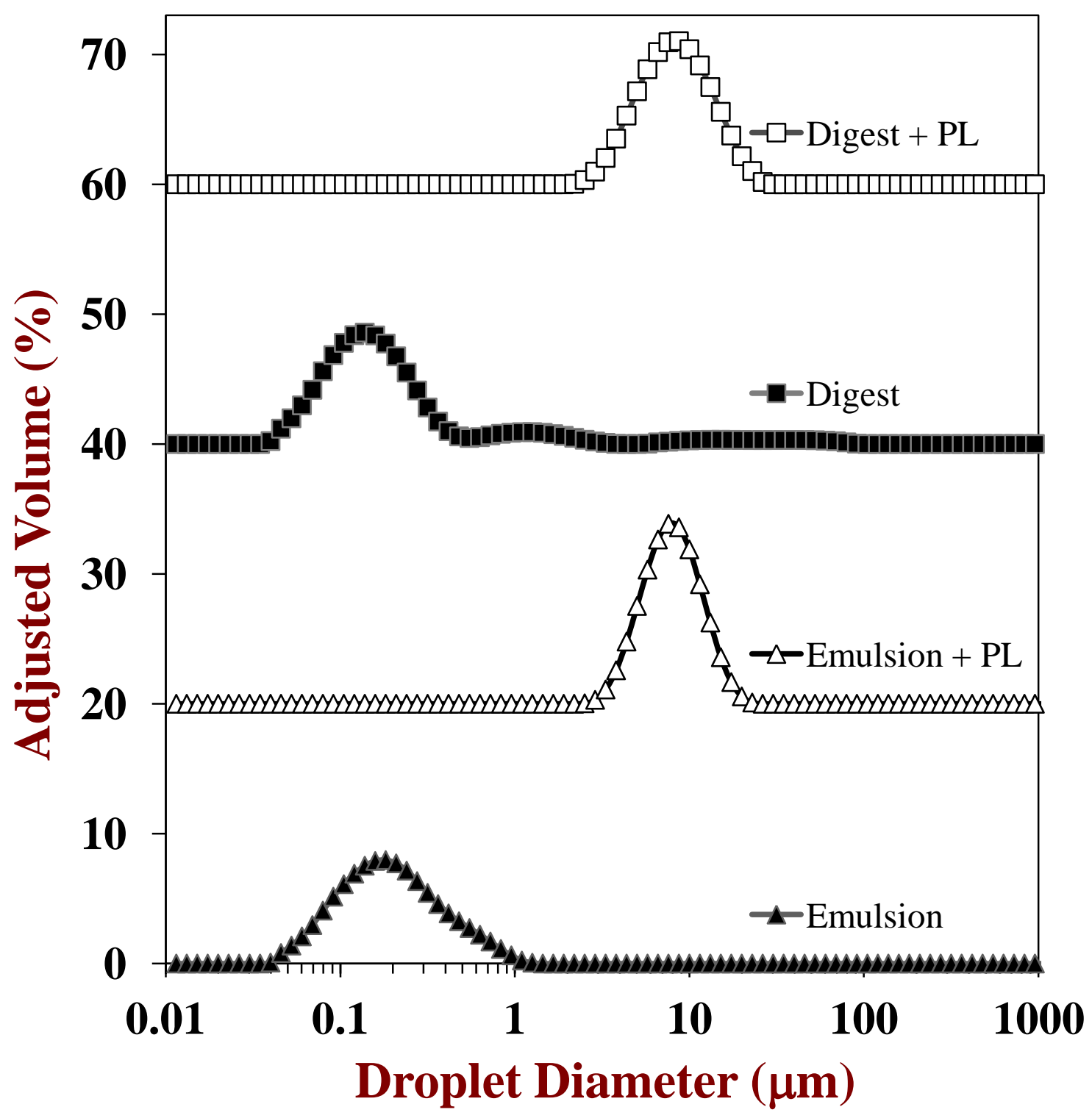

Figure 3b. Particle size distributions of the original and digested emulsion systems with and without $\varepsilon$-PL for QN-stabilized systems. 


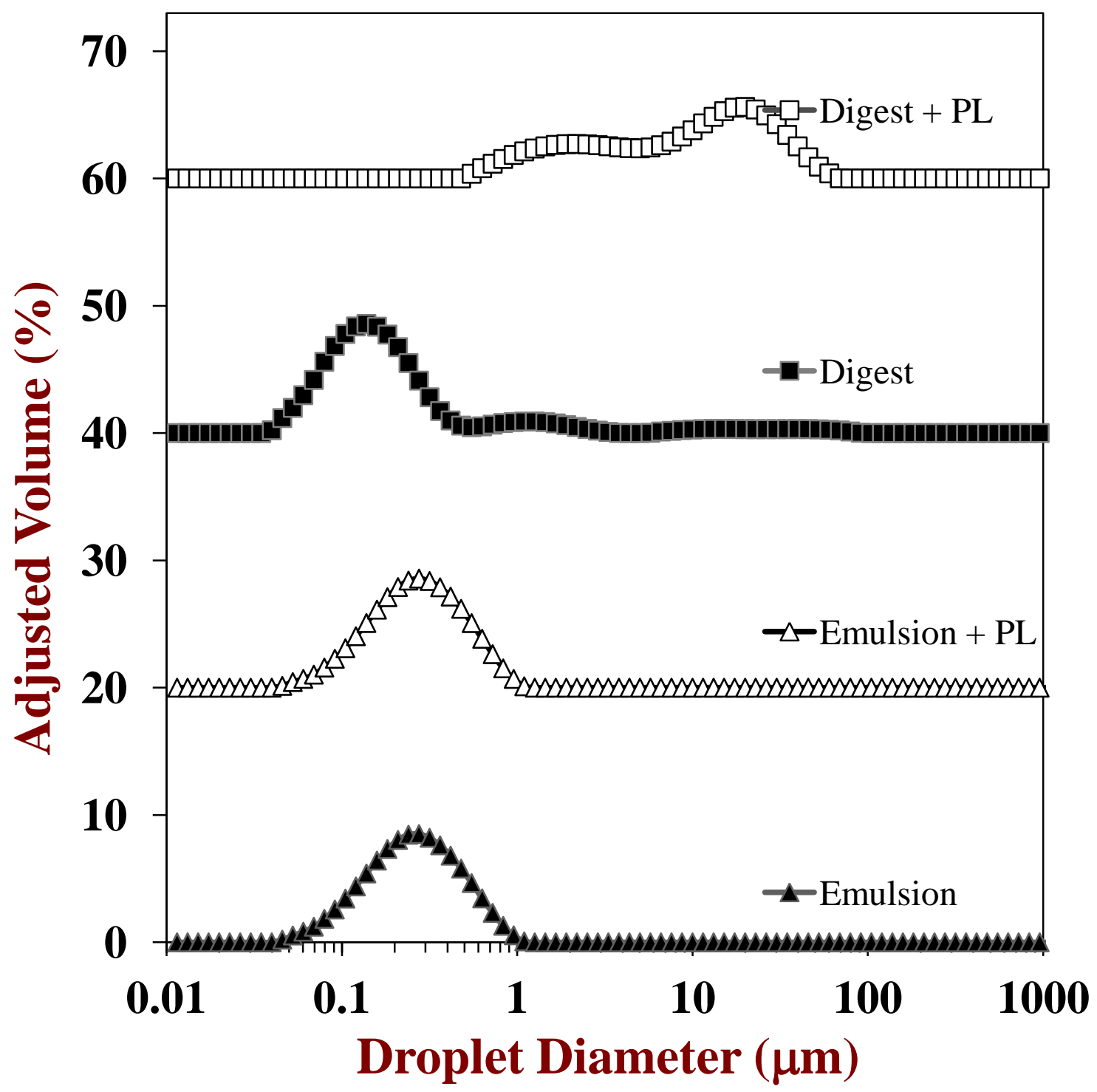

Figure 3c. Particle size distributions of the original and digested emulsion systems with and without $\varepsilon$-PL for Tween 20-stabilized systems. 


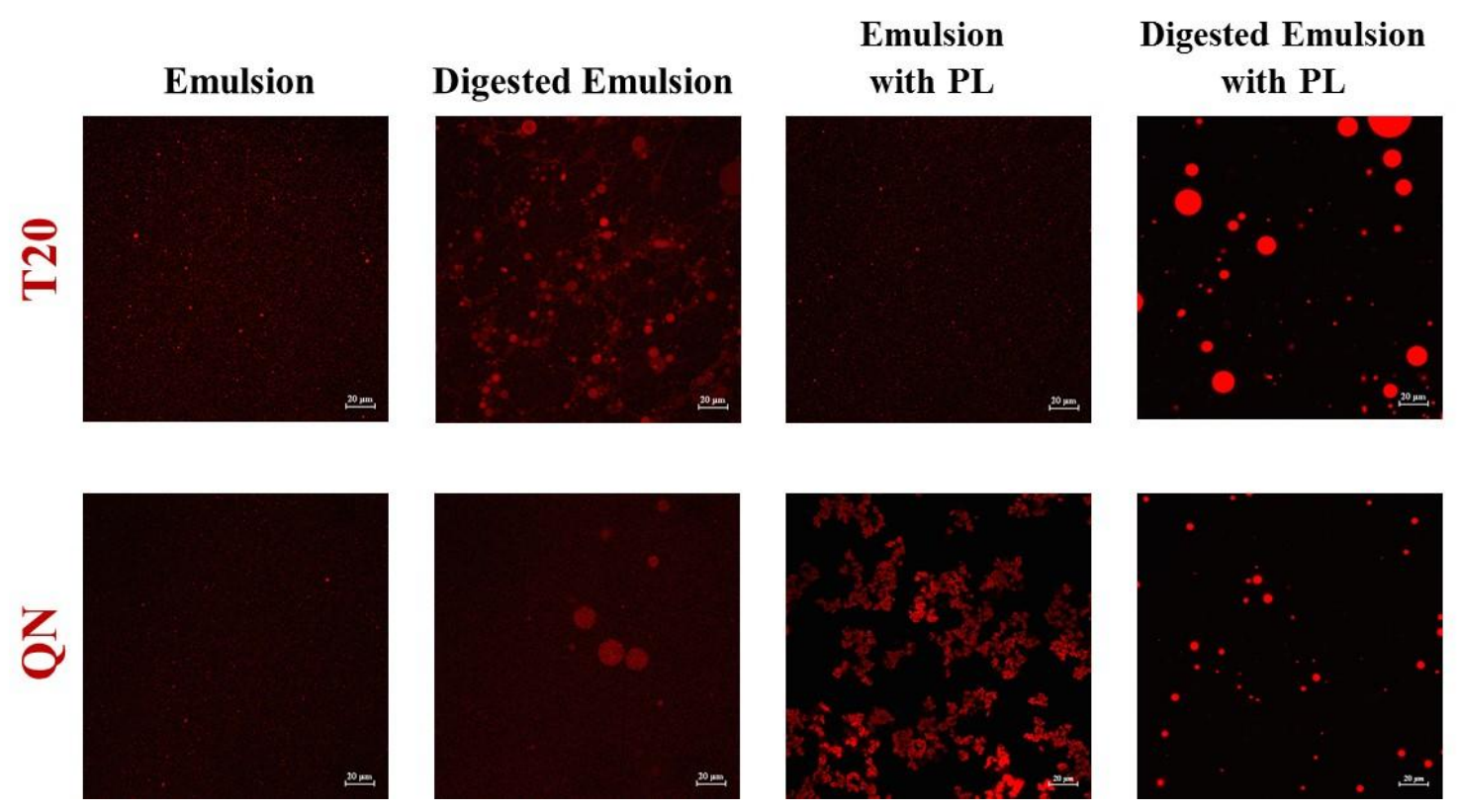

Figure 4. Images obtained via confocal microscopy of the T20- and QN-stabilized emulsions before and after digestion, with and without the addition of $\varepsilon$-PL. The scale bars are $20 \mu \mathrm{m}$ long. 


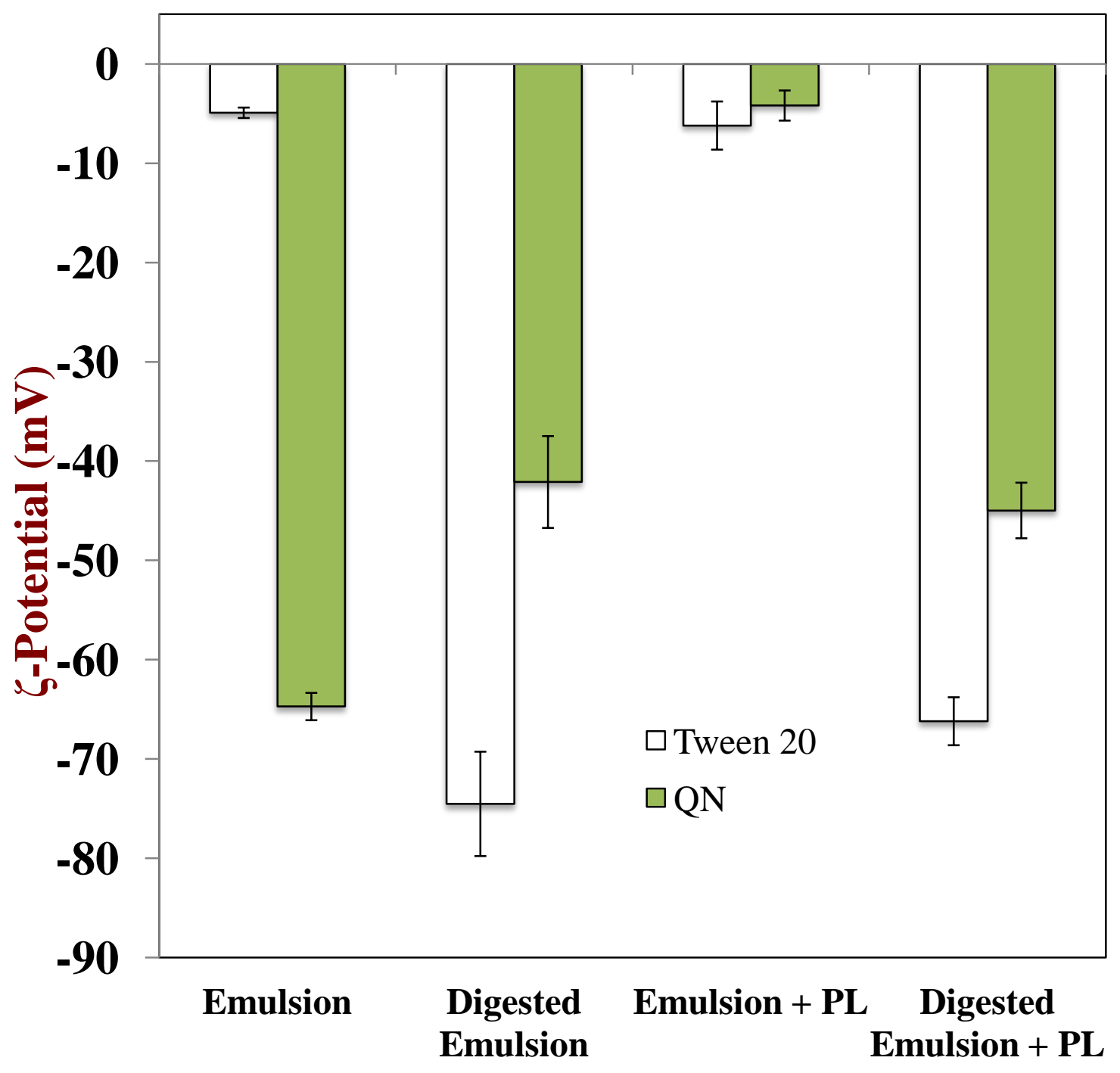

Figure 5. $\zeta$-Potential $(\mathrm{mV})$ of the original and digested nanoemulsions with and without $\varepsilon$-PL for both T20 and QN systems. 


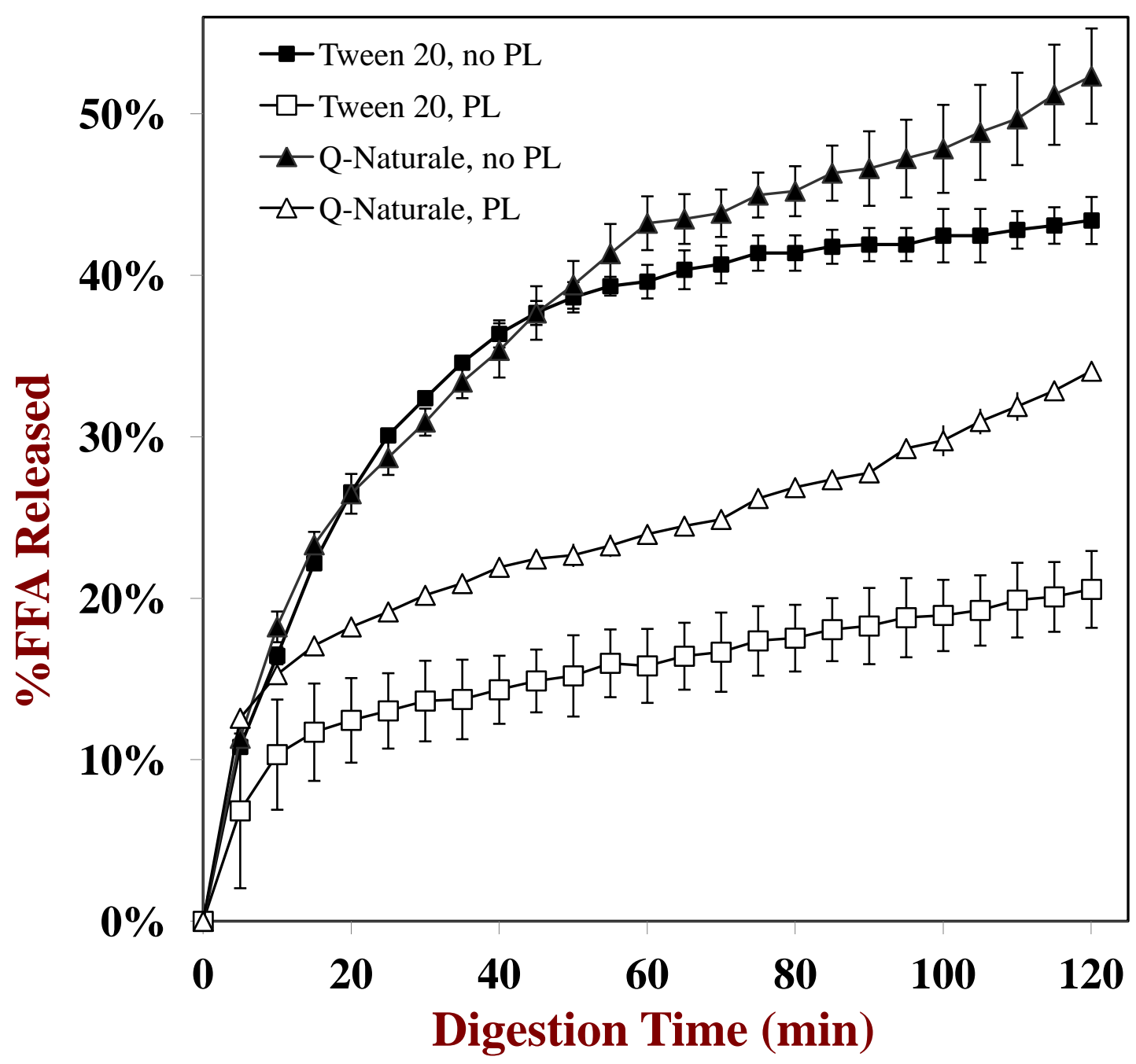

Figure 6. Influence of surfactant charge and addition of $\varepsilon$-PL on the percentage of free fatty acids (FFA\%) released from nanoemulsions formulated with anionic (QN) and neutral (T20) surfactants. The final lipid content in the digestion medium was 2.5\%. 